\title{
In vitro plantlet production of the endangered Pinguicula vulgaris
}

Tomás Grevenstuk, Anabela Romano*

Institute for Biotechnology and Bioengineering, Centre of Genomics and Biotechnology (IBB/CGB) Faculty of Sciences and Technology, University of Algarve, 8005-139 Faro, Portugal

Received 28 July 2011; Accepted 29 November 2011

Abstract: This study describes the development of a micropropagation protocol for Pinguicula vulgaris using cultures initiated from in vitro produced seedlings. P. vulgaris is a carnivorous plant with a northern, disjunctly circumpolar distribution and specific habitat requirements, and is hence becoming increasingly rare. Shoot proliferation was significantly influenced by Murashige and Skoog (MS) macronutrient concentration, showing higher proliferation rates in $1 / 4 \mathrm{MS}$, but was not affected by the addition of $0.1 \mathrm{mg} / \mathrm{L}$ 6-benzyladenine (BA) or zeatin (Zea). The best medium for propagating $P$. vulgaris was plant growth regulator (PGR) free $1 / 4 \mathrm{MSS}$. An average of 7.62 new shoots per initial explant could be obtained after 8 weeks of culture, of which over $79 \%$ produced roots during proliferation. Moreover, rooting percentages of $100 \%$ were obtained for the initial explants in all the tested media, including media without PGRs. The plantlets were successfully acclimatized to ex vitro conditions, exhibiting normal development.

Keywords: Conservation • In vitro regeneration • Plant growth regulators • Carnivorous plant.

(C) Versita Sp. z 0.0 .

\section{Abbreviations}

BA - 6-benzyladenine;

IBA - indole-3-butytic acid;

MS - Murashige and Skoog medium;

$P G R$ - plant growth regulator;

Zea - zeatin.

\section{Introduction}

Pinguicula vulgaris L. (Lentibulariaceae), or common butterwort, is a perennial carnivorous plant that thrives in nutrient-poor habitats, such as bogs and swamps, which remain sunny and moist during the growing season [1]. The species occurs in the northern and upland parts of Europe and North America, but despite its widespread distribution, $P$. vulgaris is becoming increasingly rare. Seedling establishment in the wild is precarious due to both the small seed size, which provides negligible reserves, and the scarceness of suitable wet sites free from competition by other species. Plant growth is also greatly limited by water availability, therefore, suitable habitats for $P$. vulgaris are scattered and most populations are isolated and vulnerable to extinction [2]. These main factors underline the urgent need to ensure the conservation of this species. $P$. vulgaris produces conspicuous, solitary, purple flowers, which, together with its peculiar carnivorous habit, contribute to its commercial value as an ornamental. It has also been shown recently that in vitro cultured Pinguicula plants produce the characteristic metabolites of the Pinguicula genus [3], which might be interesting for evaluation of bioactivity in drug discovery programs. In fact, leaf extracts of butterwort have been used by herbalists for their spasmolytic effects in cases of whooping cough, asthma, tuberculosis and spasms of intestinal pain [4]. Therefore, in vitro produced P. vulgaris biomass might be used for extraction of phytochemicals as well, reducing pressure on wild stocks.

The aim of this study is to develop an in vitro protocol to propagate $P$. vulgaris and to contribute to its 
preservation. The development of a micropropagation protocol for this species is of utmost importance because the establishment of a broader conservation strategy, encompassing seed cryopreservation, depends on the regeneration of plantlets in vitro.

\section{Experimental Procedures}

\subsection{Seed germination and establishment of cultures}

For culture establishment, seeds of $P$. vulgaris were collected from a population located in a protected area near Cagarouço in Peneda Gerês National Park (Trás-os-Montes e Alto Douro region, Portugal). The obtained seeds were surface sterilized by immersion in a solution of commercial bleach at $15 \%(\mathrm{v} / \mathrm{v})(5 \%$ of sodium hypochlorite) with a few drops of Tween-20 for $15 \mathrm{~min}$, and washed 3 times in sterile water. A subset of seeds was previously incubated at $5^{\circ} \mathrm{C}$ for one or two weeks, to assess the effect of cold stratification on in vitro seed germination. The seeds were then aseptically transferred into test tubes containing $10 \mathrm{~mL}$ MS (Murashige and Skoog) [5] medium with macronutrients reduced to $1 / 4$ (1/4MS) without plant growth regulators (PGRs). For all treatments, 4 replicates of 10 seeds were tested. Sucrose $(2 \%, w / v)$ was used as carbon source and media were solidified with $1 \%(\mathrm{w} / \mathrm{v})$ agar. After adding all media components, $\mathrm{pH}$ was adjusted to 5.75 with $\mathrm{NaOH}$ solution and autoclaved at $121^{\circ} \mathrm{C}$ and $1.1 \mathrm{Kg} / \mathrm{cm}$ for $20 \mathrm{~min}$. All cultures were incubated under a $16 \mathrm{~h}$ photoperiod provided by cool-white fluorescent lights at a photon flux density of $60 \mathrm{mmol} \mathrm{m}^{-2} \mathrm{~s}^{-1}$ and at $25 \pm 2^{\circ} \mathrm{C}$.

\subsection{Proliferation and rooting}

Two months after germination roots were discarded and the entire shoot was sub-cultured onto fresh $1 / 4 \mathrm{MS}$ medium in order to obtain sufficient number of shoots for the subsequent assays. After 8 weeks, shoots with identical size were separated and used in the different assays. The effect on shoot proliferation of two MS macronutrient concentrations (MS and $1 / 4 \mathrm{MS}$ medium) and of two cytokinins [6-benzyladenine (BA) and zeatin (Zea) at $0.1 \mathrm{mg} / \mathrm{L}$ ] in $1 / 4 \mathrm{MS}$ medium was evaluated. The combination of the cytokinins BA and Zea, at $0.1 \mathrm{mg} / \mathrm{L}$, with the auxin indole-3-butyric acid (IBA), at $0.01 \mathrm{mg} / \mathrm{L}$, in $1 / 4 \mathrm{MS}$ basal medium was also assessed. Since after 8 weeks of culture simultaneous proliferation and rooting was observed in all tested media, both proliferation and rooting results were recorded. Ten shoots were inoculated per assay and four replicates were tested for each medium. The proliferation capacity was evaluated by the proliferation frequency, which represents the ability of each explant to regenerate new shoots, and the proliferation rate, which was assessed as the mean number of shoots produced by each explant at the end of the subculture period. Since the shoots grew like a small horizontal rosette, it was not possible to evaluate shoot length. Rooting capacity was expressed in terms of rooting percentage of initial explants and new shoots, root number and the longest root length per plantlet.

\subsection{Plantlet acclimatization}

Plantlets with at least 4 roots longer than $10 \mathrm{~mm}$ were selected, removed from the culture flasks and the roots cleared of culture media to prevent pathogenic contamination during acclimatization ex vitro. The plantlets were placed in $100 \mathrm{~mL}$ plastic pots containing a mixture of peat and vermiculite $(3: 1, v / v)$ and acclimatised inside transparent polyethylene boxes. The plantlets were maintained in a growth room for 3 weeks under controlled conditions ( $16 \mathrm{~h}$ photoperiod, $60 \mathrm{mmol} \mathrm{m}^{-2} \mathrm{~s}^{-1}$ and $25 \pm 2^{\circ} \mathrm{C}$ ), and then gradually exposed to reduced relative humidity by progressively opening the plastic covers over a period of 3 weeks.

\subsection{Statistical analysis}

The data were subjected to analysis of variance to assess treatment differences using the SPSS statistical package for Windows (release 15.0, SPSS INC.). Significance between means was tested by Duncan's New Multiple Range Test $(P=0.05)$.

\section{Results and Discussion}

\subsection{Seed germination}

Many carnivorous plant species need a cold treatment to break dormancy $[6,7]$, however the tested cold treatments did not influence the seed germination. The attained germination rates were very low and no large differences were observed between the control (24.24\%) and cold treatments (1 week: $25.64 \%$; 2 weeks: $24.14 \%)$. These results confirm previous findings by Heslop-Harrison [8], who observed that vernalization does not promote germination. However, the author reported germination rates of $100 \%$ for both control and cold stratification treatments. These findings suggest that the low germination percentages were due to low seed viability. Seeds are the preferred starting material for establishing cultures of rare species as this ensures that a wide genetic base is maintained [9]. Moreover, the use of seeds for the establishment of primary cultures can prevent most of the contamination problems that are often associated with explant establishment. In fact, in our experiment 
all seeds were free from contaminations after the applied sterilization procedure (Figure $1 \mathrm{~A}$ ).

\subsection{Shoot proliferation and rooting}

The development of a micropropagation protocol for $P$. vulgaris proved to be a difficult task in comparison to other species of the genus [10]. Due to their delicate nature, cultures were very susceptible to the separation step at the beginning of each assay. Explants dried out or lost viability during the course of the 8 weeks culture period when excision was not performed accurately. In contrast, $P$. lusitanica was efficiently micropropagated by our group in $1 / 2 \mathrm{MS}$ medium supplemented with $\mathrm{BA}$ at $0.5 \mathrm{mg} / \mathrm{L}$, with high proliferation rates (over 28 shoots per initial explant) and rooting percentages of $100 \%$ [10]. A study performed by Clapa et al. [11] showed that high multiplication rates and vigorous $P$. vulgaris plantlets could be obtained if longer subcultures periods are used.

Due to the limiting amount of plant material, the number of planned assays was considerably reduced. Initially, the influence of two macronutrient concentrations, MS and $1 / 4 \mathrm{MS}$, was evaluated and it was observed that higher macronutrient concentration negatively effected most of the growth parameters (Figure 2). The shoot proliferation frequency obtained in MS medium (63.33 $\pm 3.33 \%$; Figure $2 \mathrm{~A}$ ) was lower than in $1 / 4 \mathrm{MS}(90.00 \pm 5.77 \%)(P<0.05)$. The same was observed for the rooting percentage of the new formed shoots (Figure 2D: $56.87 \pm 3.44 \%$ in $\mathrm{MS}$ and $79.74 \pm 1.35 \%$ in $1 / 4 \mathrm{MS}$ ), the number of roots of the initial explant (Figure 2E: $3.22 \pm 0.28$ in $\mathrm{MS}$ and $5.11 \pm 0.24$ in $1 / 4 \mathrm{MS}$ ), and the longest root length (Figure $2 \mathrm{~F}: 8.83 \pm 0.77 \mathrm{~mm}$ in $\mathrm{MS}$ and $13.54 \pm 0.82 \mathrm{~mm}$ in $1 / 4 \mathrm{MS})(P<0.05)$. Despite the differences not being statistically significant, the number of shoots produced per initial explant (Figure 2B) was higher in $1 / 4 \mathrm{MS}$ medium $(5.06 \pm 0.61$ in MS and $7.62 \pm 0.51$ in $1 / 4 \mathrm{MS}$ ). These results are in agreement with those obtained for other carnivorous plants in which shoot proliferation was promoted in low macronutrient concentration media $[10,12,13]$. The preference of carnivorous plants for media with a low concentration of macronutrients may be related with the adaptation to their natural habitats, as carnivorous plants usually subsist in nutrient poor soils.

The subsequent assays were thus performed in $1 / 4 \mathrm{MS}$ basal media, supplemented with the cytokinins
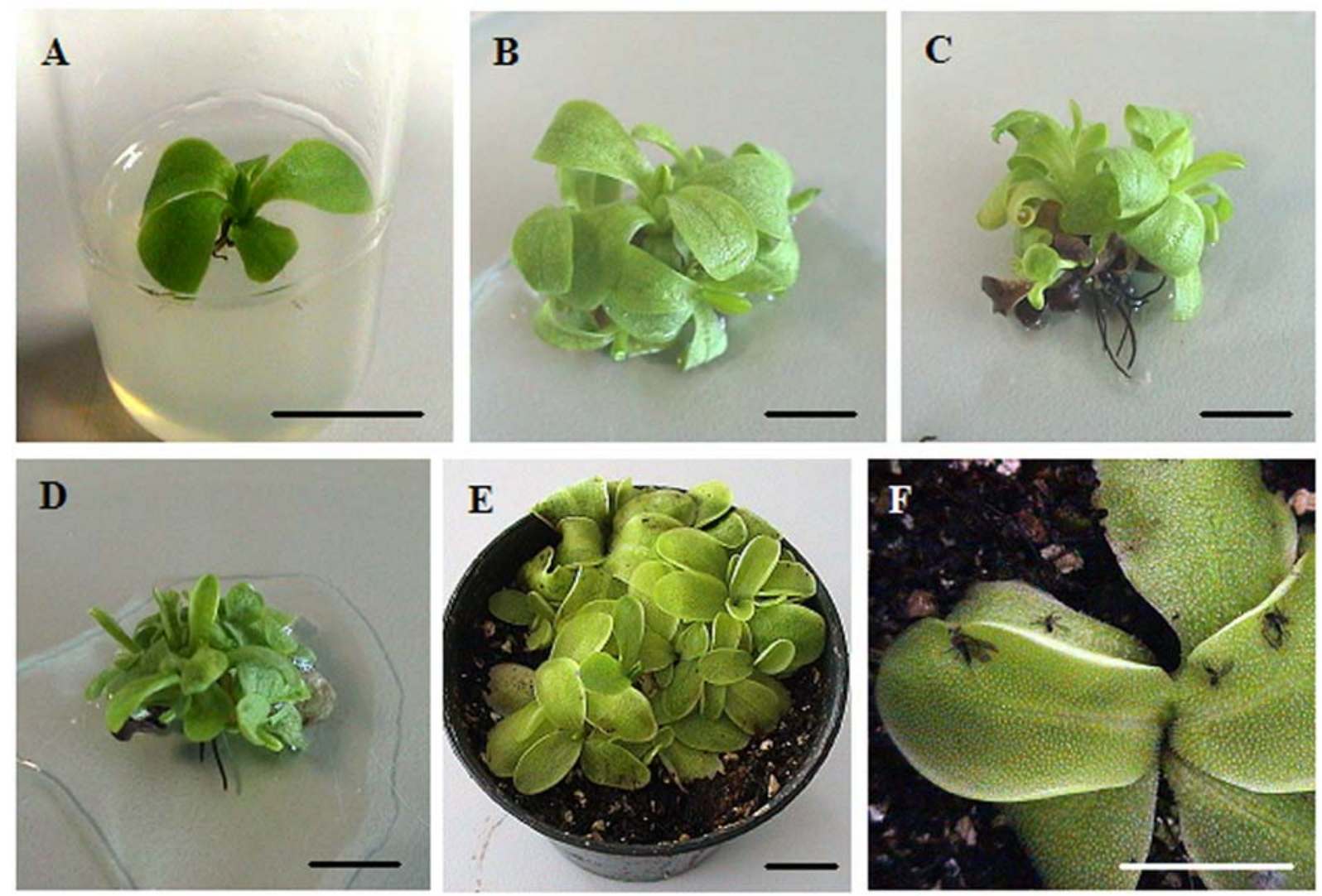

Figure 1. Micropropagation of $P$. vulgaris: seedling explants used in the assays (A); shoots at the end of proliferation phase in 1/4MS without PGR (B); supplemented with BA at $0.1 \mathrm{mg} / \mathrm{L}(\mathrm{C})$ or with Zea at $0.1 \mathrm{mg} / \mathrm{L}(\mathrm{D})$; acclimatized plants with 2 months in ex vitro conditions (E); leaf detail of acclimatized plant with captured insects $(F)$. Bars $=1 \mathrm{~cm}$. 


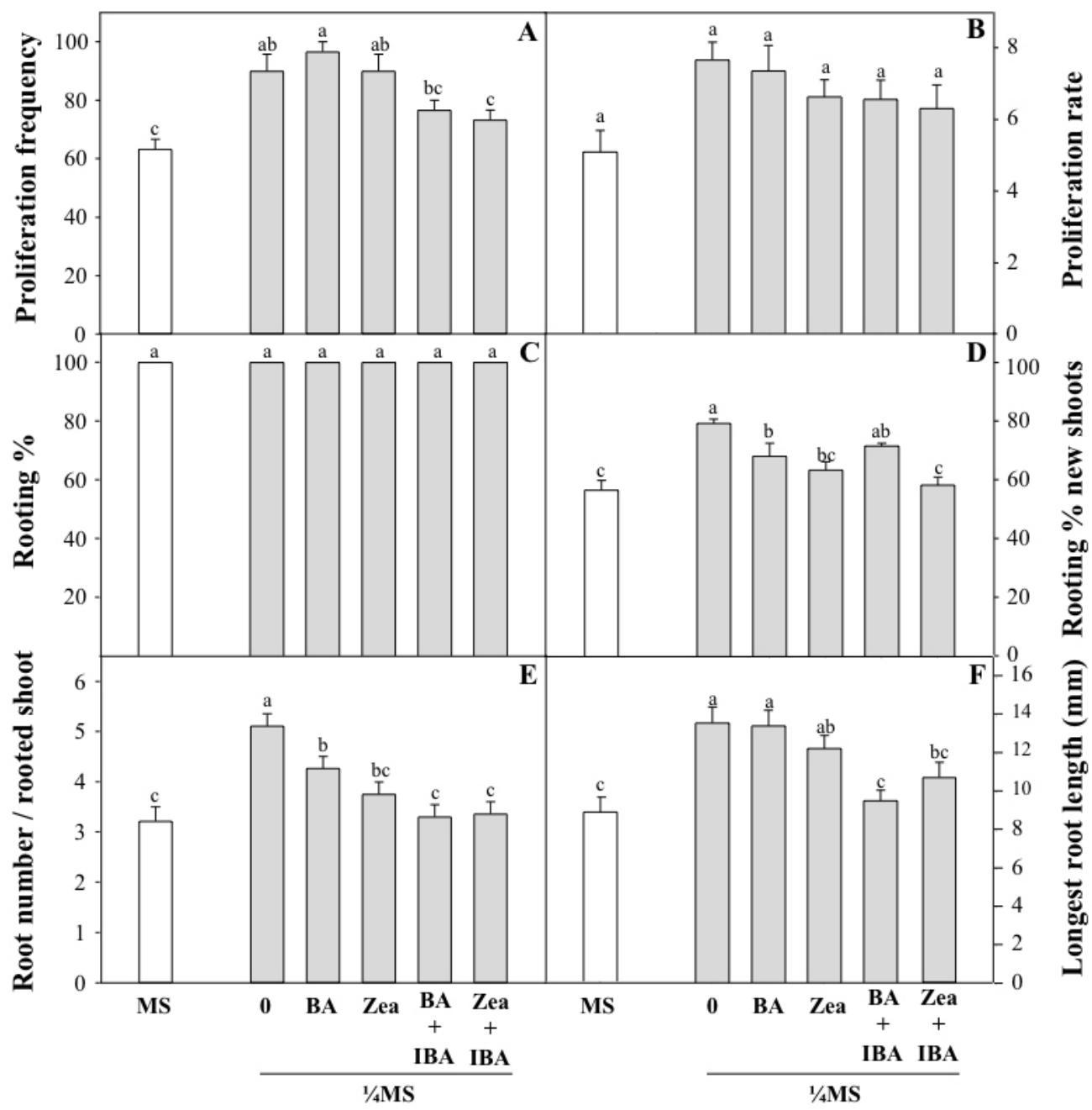

Figure 2. Effect of MS medium concentration, cytokinin type (BA and Zea at $0.1 \mathrm{mg} / \mathrm{L}$ ) and combination of cytokinin (0.1 mg/L) and auxin (IBA at $0.01 \mathrm{mg} / \mathrm{L}$ ) on proliferation and rooting of $P$. vulgaris shoots: proliferation frequency (A), proliferation rate (B), rooting percentage of initial explant $(C)$, rooting percentage of newly produced shoots $(D)$, mean number of developed roots per initial explant $(E)$, longest root length of initial shoot (F). Values represent means \pm SE of 4 replications with 10 shoots. In each graph columns with different letters are significantly different at $P<0.05$ according to Duncan's multiple range test.

BA or Zea at $0.1 \mathrm{mg} / \mathrm{L}$, since preliminary assays pointed out that higher concentrations did not promote proliferation (data not shown). The results show no significant differences between treatments regarding the proliferation frequency (Figure $2 \mathrm{~A}$ ), proliferation rate (Figure $2 \mathrm{~B}$ ) and rooting percentage of the initial explant (Figure 2C) $(P \geq 0.05)$. The proliferation frequency was on average above $90 \%$, the number of produced shoots over 6.60 , and the rooting percentage of the initial explant was $100 \%$ in all the assays. Noteworthy, all shoots grown in the other tested media rooted as well, independently of the macronutrient concentration or
PGR supplementation (Figure 2C). The proliferation rate was not influenced by the macronutrient concentration of the basal media or by the addition of cytokinins (Figure 2B) $(P \geq 0.05)$. However, in the specific case of this species, the conclusions drawn from the statistical analysis do not reflect the discrepancies observed from a morphological perspective. In general, the cultures grown in PGR-free medium produced vigorous and healthy looking shoots, while the cultures grown in cytokinin-supplemented media frequently displayed signs of necrosis, underdevelopment and curdled leaves with a brittle texture, especially in the case of shoots 
grown in Zea containing medium (Figure 1B,C,D). In fact, proliferation and rooting parameters alone do not always reflect the real efficiency of the experimental conditions. As stated before, no statistical differences were observed between shoots cultured in PGR-free and cytokinin-supplemented medium after the first subculture. However, shoots cultured in cytokininsupplemented medium have little viability and therefore cannot be used for subsequent subculturing. This is an essential issue considering that it is not only important to produce a large amount of new shoots, but also to produce shoots capable of regenerating new vigorous plantlets.

Besides the previously cited works [10,11], the only micropropagation protocol published for Pinguicula is the one developed by Adams et al. [14] for $P$. moranensis H.B.K. The author used leaf explants as starting material and found that the highest plantlet number and growth rate were obtained using $1 / 5$ Linsmaier-Skoog medium supplemented with a combination of $B A(0.02 \mathrm{mg} / \mathrm{L})$ and 2-naphthaleneacetic acid (0.01-0.10 mg/L). Although several authors have reported a synergistic effect between cytokinins and auxins during shoot proliferation $[15,16]$, in this work culture media combining BA or Zea $(0.1 \mathrm{mg} / \mathrm{L})$ with IBA $(0.01 \mathrm{mg} / \mathrm{L})$ were not effective in enhancing proliferation $(P \geq 0.05)$ (Figure 2$)$, which is in good agreement with the results obtained for P. Iusitanica [10].

As noted before, the results presented in Figure $2 \mathrm{C}$ and $D$ show that $P$. vulgaris shoots have a great predisposition for rooting. In fact, initial explants as well as the new produced shoots rooted spontaneously without the addition of auxins. Independently of the growth media, more than $50 \%$ of the new shoots were able to produce roots. Shoots in an early developmental stage did not produce roots, suggesting that root formation is more dependent on shoot developmental stage than on the media composition. The rooting of $P$. vulgaris shoots without addition of auxins was also reported by Clapa et al. [11] and despite being unclear why this species does not require auxins for rooting, the obtained results could be due to high levels of endogenous auxins in shoots, as previously reported in other species [17]. This suggests that the development of a more efficient protocol should be directed to obtain a larger number of shoots, without compromising shoot viability, since the rooting phase does not have to be optimized. Plantlets with well-developed roots were successfully acclimatized and the leaves of the micropropagated plants were functional and able to catch insects (Figure 1E,F).

\section{Conclusions}

This study reports an efficient protocol to regenerate $P$. vulgaris plantlets in vitro using seedlings as explants. The best medium was found to be $1 / 4 M S$ PGR-free medium, as it provided the highest proliferation and rooting response. The described protocol allows $P$. vulgaris to be maintained in an in vivo germplasm collection and to regenerate plantlets that can be used to replenish declining natural populations, making a valuable contribution to the conservation of this species. In addition, the described protocol can be integrated in a broad conservation project based on seed cryopreservation, for which an in vitro regeneration procedure is fundamental.

\section{Acknowledgements}

The authors would like to thank Dr. Henrique Pereira of the Institute for Nature Conservation and Biodiversity (ICNB) for providing P. vulgaris seeds. T. Grevenstuk acknowledges a grant from the Portuguese Science and Technology Foundation (FCT, Grant SFRH/ BPD/73293/2010).

\section{References}

[1] Blanca G., Ruiz-Rejón M., Zamora R., Taxonomic revision of the genus Pinguicula in the Iberian Peninsula, Folia Geobot., 1999, 34, 337-361

[2] Zamora R., Jamilena M., Rejón M.R., Gabriel Blanca G., Two new species of the carnivorous genus Pinguicula, (Lentibulariaceae) from Mediterranean habitats, Plant Syst. Evol., 1996, 200, 41-60

[3] Grevenstuk T., van der Hooft J., Vervoort J., Romano A., Iridoids and caffeoyl phenylethanoid glucosides from Pinguicula lusitanica (Lentibulariaceae), Biochem. Syst. Ecolog., 2009, 37, 285-289

[4] Christen K., [Beitrag zur Pharmakochemie und Pharmakologie des gemeinen Fettkrautes (Pinguicula vulgaris L.)], Die Pharmazie Arzneipflanzen-umschau, 1961, 11, 92-102 (in German)

[5] Murashige T., Skoog F., A revised medium for rapid growth and bioassays with tobacco tissue cultures, Physiol. Plant., 1962, 15, 473-497 
[6] Jang G.-W., Kim K.-S., Park R.-D., Micropropagation of Venus fly trap by shoot culture, Plant Cell Tiss. Org. Cult., 2003, 72, 95-98

[7] Jayaram K., Prasad M.N.V., Drosera indica L. and D. burmanii Vahl., medicinally important insectivorous plants in Andhra Pradesh - regional threats and conservation, Curr. Sci., 2006, 91, 943-946

[8] Heslop-Harrison Y., Pinguicula L, J. Ecol., 2004, 92, 1071-118

[9] Benson E.E., Danaher J.E., Pimbley I.M., Anderson C.T., Wake J.E., Daley S., et al., In vitro micropropagation of Primula scotica: a rare Scottish plant, Biodivers. Conserv., 2000, 9, 711-726

[10] Gonçalves S., Escapa A.L., Grevenstuk T., Romano A., An efficient in vitro propagation system for Pinguicula lusitanica, a rare insectivorous plant, Plant Cell Tiss. Org. Cult. 2008, 95, 239-243

[11] Clapa D., Fira A., Pacurar I., In vitro propagation of Pinguicula vulgaris, Bulletin UASVM Horticulture, 2010, 67, 330-335

[12] Kim K.-S., Jang G.-W., Micropropagation of Drosera peltata, a tuberous sundew, by shoot tip culture, Plant Cell Tiss. Org. Cult., 2004, 77, 211-214

[13] Grevenstuk T., Coelho N., Gonçalves S., Romano A., In vitro propagation of Drosera intermedia in a single step, Biol Plantarum, 2010, 54, 391-394

[14] Adams R.M., Koenigsberg S.S., Langhans R.W., In vitro propagation of the Butterwort Pinguicula moranensis H.B.K., HortScience, 1979, 14,701 $-702$

[15] Skiada F.G., Grigoriadou K., Eleftheriou E.P., Micropropagation of Vitis vinifera L. cv. 'Malagouzia' and 'Xinomavro', Cent. Eur. J. Biol., 2010, 5, 839852

[16] Cantos M., Linán J., García J.L., García-Linán M., Domínguez M.A., Troncoso, A., The use of in vitro culture to improve the propagation of Rhododendron ponticum subsp. baeticum (Boiss. \& Reuter), Cent. Eur. J. Biol., 2007, 2, 297-306

[17] Malá J., Gaudinová A., Dobrev P., Eder J., Cvikrová M., Role of phytohormones in organogenic ability of elm multiplicated shoots, Biol. Plantarum, 2005, 50, 8-14 